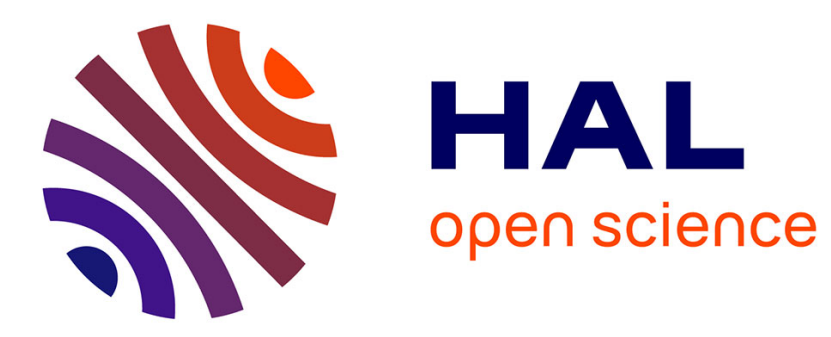

\title{
Évaluation dentaire avant une chirurgie orthopédique: revue systématique de la littérature
}

Sophie Barrere, Nicolas Reina, Ove A. Peters, Lucie Rapp, Jean-Noel Vergnes, Delphine Maret

\section{To cite this version:}

Sophie Barrere, Nicolas Reina, Ove A. Peters, Lucie Rapp, Jean-Noel Vergnes, et al.. Évaluation dentaire avant une chirurgie orthopédique: revue systématique de la littérature. Revue de Chirurgie Orthopédique et Traumatologique, 2019, 105, pp.506 - 517. 10.1016/j.rcot.2019.04.020 . hal-03484354

\section{HAL Id: hal-03484354 https://hal.science/hal-03484354}

Submitted on 20 Dec 2021

HAL is a multi-disciplinary open access archive for the deposit and dissemination of scientific research documents, whether they are published or not. The documents may come from teaching and research institutions in France or abroad, or from public or private research centers.
L'archive ouverte pluridisciplinaire HAL, est destinée au dépôt et à la diffusion de documents scientifiques de niveau recherche, publiés ou non, émanant des établissements d'enseignement et de recherche français ou étrangers, des laboratoires publics ou privés.

\section{다)(1) $(5$}

Distributed under a Creative Commons Attribution - NonCommerciall 4.0 International 
Evaluation dentaire avant une chirurgie orthopédique : Revue systématique de la littérature Dental Assessment Prior to Orthopedic Surgery: A Systematic Review

Sophie Barrere ${ }^{a}$, Nicolas Reina ${ }^{\text {b,c }}$, Ove A. Peters ${ }^{\text {d, Lucie Rapp }}{ }^{\text {a }}$, Jean-Noel Vergnes ${ }^{\text {a,e }}$, Delphine Maret $^{\text {a,c,* }}$.

a Dental Faculty, Paul Sabatier University, Toulouse University Hospital (CHU de Toulouse), 3 chemin des Maraichers, 31400 Toulouse, France.

b Department of Orthopedic Surgery, Hôpital Pierre-Paul-Riquet, CHU de Toulouse, Place du Dr Baylac TSA 4003131059 Toulouse cedex 9, France

c AMIS Laboratory - Laboratoire Anthropologie Moléculaire et Imagerie de Synthèse, Université de Toulouse, UMR 5288 CNRS, UPS, 37 allée Jules Guesde 31000, Toulouse, France.

d Department of Endodontics, Arthur A. Dugoni School of Dentistry University of the Pacific, 2155 Webster St \# 615, San Francisco, CA 94115, California, USA

e Division of Oral Health and Society, Faculty of dentistry, McGill University, 2001 McGill College, Montreal, Quebec, H3A1G1 Canada.

* Corresponding author: Delphine Maret, Dental Faculty, Paul Sabatier University, Toulouse University Hospital (CHU de Toulouse), France.

E-mail address: comtesse-maret.d@chu-toulouse.fr

Ne pas utiliser, pour citation, la référence française de cet article, mais celle de l'article original paru dans Orthopaedics \& Traumatology: Surgery \& Research, en utilisant le DOI ci-dessus. 


\section{Résumé}

\section{Contexte:}

Afin de réduire le risque d'infection après une chirurgie orthopédique, les patients doivent effectuer différents bilans médicaux préopératoires. Cependant, à notre connaissance, il n'existe aucune revue systématique de la littérature pour évaluer l'efficacité du bilan buccodentaire avant une chirurgie orthopédique. Nos problématiques sont les suivantes: 1) Y a-t-il un lien entre la présence d'un bilan dentaire préopératoire et les infections orthopédiques; 2) La présence de facteurs de risques et comorbidités augmente-elle la probabilité de survenue d'une infection orthopédique?

\section{Matériels et Méthodes:}

Les recherches ont été effectuées sur les bases de données de PubMed, Cochrane Library databases et Google Scholar sur des articles en anglais jusqu'en novembre 2018. Les critères d'inclusion sont la description d'une infection orthopédique et d'une infection dentaire, ainsi qu'une potentielle origine dentaire des agents infectieux. Les études traitant d'une évaluation dentaire effectuée avant la chirurgie sont inclues.

\section{Résultats:}

Basées sur nos critères d'éligibilités, 12 séries de cas, 4 études cas-témoins and 12 études de cohortes ont été inclues. Pour les cas-témoins, les infections de prothèses articulaires sont supposées associées à des abcès dentaires dans 6/224 cas (2.9\%). Dans les études de cohortes, l'exposition est définie comme "tout bilan ou traitement dentaire effectué avant la chirurgie". Même si seulement 4 études de cohortes fournissent cette information, il semble que la présence d'une complication infectieuse soit moins fréquente si l'examen dentaire préopératoire est effectué. 
Les traitements dentaires réalisés avant la chirurgie sont principalement des détartragessurfaçages pour 78/205 cas (38\%), des avulsions pour 49/205 cas (24\%) et des soins conservateurs pour $37 / 205$ cas $(18 \%)$.

\section{Discussion:}

L'hétérogénéité des études inclues dans cette revue de la littérature ne permet pas de conclure sur la nécessité de ce bilan. Bien qu'il n'y ait aucune preuve scientifique pour ou contre le bilan dentaire préopératoire avant chirurgie orthopédique, il est conseillé d'effectuer ce bilan afin de maintenir une hygiène dentaire favorable et réduire les facteurs de risque.

Niveau de preuve : III, revue systématique

Mots clés : infections dentaires; infections articulaires periprothétiques ; évaluation dentaire

\section{Introduction}

L'infection de prothèses articulaires est la complication la plus dévastatrice pour les patients porteurs de prothèses totales [1]. L'incidence des infections de prothèses articulaires varie de 0.8 à $1.9 \%$ après la pose d'une prothèse primaire totale de genou [2-4] et de 0.3 to $1.7 \%$ après la pose d'une prothèse totale de hanche [4-8]. Plusieurs facteurs de risque d'infection de prothèses articulaires sont décrits dans la littérature. Les facteurs liés à l'hôte incluent une antériorité chirurgicale ou une précédente infection associée à une prothèse articulaire sur le même site, le tabac, l'obésité, l'arthrose rhumatoïde, des néoplasmes, une immunosupression ou un diabète [9-12]. Les facteurs de risque post-opératoire incluent les complications de cicatrisation (e.g, infection superficielle, hématome après cicatrisation, nécrose de la plaie et 
déhiscence), fibrillation atriale, infarctus du myocarde, infection urinaire, prolongation de l'hospitalisation et bactériémie au Staphylococcus aureus [2-5, 9-19]. Dans le but de réduire le risque d'infection après une chirurgie orthopédique, il est demandé aux patients d'effectuer des bilans préopératoires dans différents domaines médicaux. La recherche de foyers infectieux à distance est également recommandée car les bactériémies associées à des infections aigues dentaires, de la peau, et des tractus respiratoire gastro-intestinal et urogénital sont connues pour causer des infections périprothétiques [20-22].

L'infection focale d'origine dentaire peut être définie comme une infection survenant dans différents tissus ou organes du corps, causées par des micro-organismes (ou leurs produits) présents dans la cavité buccale [23]. L'incidence des infections focales d'origine dentaire est de l'ordre de 0.03 à $0.04 \%$ [24,25]. La bactériémie est le mécanisme d'infection focale le plus connu et peut intervenir spontanément (par le brossage des dents, la mastication) ou peut être causée par des traitements dentaires (e.g. détartrage, traitement endodontique, avulsions) [26,27], ce qui soulève la question de la prescription d'une antibioprophylaxie avant de réaliser des traitements dentaires. [28-30]. Actuellement, l'antibioprophylaxie pour des chirurgies orales est déconseillée [29-31]. En effet, le coût et les risques associés sont disproportionnés vis à vis de l'efficacité. [29]. Cependant, lors de la période préopératoire, le chirurgien orthopédique demande habituellement un certificat déclarant que les foyers infectieux potentiels du patient ont été contrôlés et éliminés. La question de comment effectuer ce bilan préopératoire reste incertaine.

Nous avions pour but de faire une revue systématique et de synthétiser toute la littérature concernant la réalisation de l'évaluation bucco-dentaire avant une chirurgie orthopédique. Le but de cette revue de la littérature est de répondre aux deux questions suivantes: 1) Y a-t-il un lien entre la présence d'une évaluation dentaire préopératoire et les 
infections orthopédiques? 2) La présence de facteurs de risques et comorbidités augmente-elle la probabilité de survenue d'une infection orthopédique?

\section{Stratégie et critères de recherche}

\subsection{Sources de données et recherche}

Nous avons suivi les directives PRISMA pour planifier et conduire cette revue systématique. [32]. L'approbation éthique n'est pas nécessaire pour notre étude car c'est une revue systématique de la littérature existante et donc n'implique pas la manipulation de données personnelles de patients.

Les recherches documentaires d'articles en anglais ont été réalisées jusqu'en novembre 2018 sur les bases de données de PubMed, Cochrane Library et Google Scholar. Aucune limite de date de publication n'a été appliquée. La stratégie de recherche était la suivante: [“odontology" OR "dental infection" OR "sites of infection" OR "dental assessment”] AND ["infection of joint prosthesis" OR "hematogenous infection" OR "dental focal infection" OR “joint prosthesis”]. Pour chacun des mots clés nous avons utilisé un descripteur du MeSH. Une recherche manuelle complète la recherche électronique afin de trouver d'éventuelles études supplémentaires dans les références bibliographiques de chaque article pertinent.

\subsection{Sélection des articles}

Une première sélection a été réalisée, basée sur le titre et le résumé des études trouvées lors de nos recherches (Figure 1). Les critères d'inclusions pour la sélection des articles étaient:

1) Les études traitant du bilan bucco-dentaire avant une chirurgie orthopédique ;

2) Les études où le pathogène retrouvé pouvait être d'origine dentaire; et

3) Les études décrivant des infections de prothèses articulaires et des infections dentaires Une fois les articles complets récupérés, nous avons exclus tous ceux traitant des sujets suivants: 
1) Les infections hématogènes causées par des traitements dentaires sans évaluation des prothèses articulaires. Les études qui décrivent une bactériémie causée par un traitement dentaire comme des avulsions ou des traitements parodontaux. Cependant, pour évaluer la relation entre infections dentaires et infections de prothèses articulaires, nous nous sommes concentrés uniquement sur les infections dentaires présentent avant la chirurgie orthopédique.

2) Les infections superficielles de la plaie, puisque les infections qui interviennent immédiatement après la chirurgie concernent le tissus sous cutané, alors que les infections de prothèses articulaires causées par une infection dentaire sont hématogènes.

3) Les éditoriaux et les recommandations.

4) Les études dont le suivi s'est arrêté au moment de la pose de la prothèse.

\subsection{Extraction des données}

Deux auteurs indépendamment (SB et JNV) ont scanné les titres et résumés des articles potentiels. La décision finale sur la pertinence des articles n'a été prise qu'après un examen plus approfondi des textes complets. Les données suivantes ont été extraites des articles: type d'étude (i.e. essai clinique, séries de cas, cas-témoins, cohorte ou étude croisée), nombre de participants, âge et proportion homme-femme, type de prothèse placée, présence du bilan dentaire ou non, pathogène impliqué (si renseigné), site de l'infection orale suspectée, comorbidités associées.

\subsection{Evaluation qualitative}

Nous avons réalisé une évaluation qualitative de chaque article, en utilisant le NewcastleOttawa scale (NOS) pour les études cas témoins et cohortes [33].

\section{Résultats}

\subsection{Résultats des recherches}


Un total de 84 articles a été sélectionné dans les bases de données, dont 28 articles éligibles pour cette revue (Figure 1). Douze articles sont des séries de cas, quatre sont des études cas témoins, et douze sont des études de cohorte. Aucun essai clinique n'est axé sur l'examen ou les traitements dentaires en cas d'infection de prothèses articulaires.

\subsection{Evaluation dentaire préopératoire}

Dans les séries de cas, seulement un article a renseigné la présence de l'examen dentaire préopératoire. Pour tous les articles, les pathologies orales à distance impliquées étaient des parodontites pour 8/29 cas (27\%), des lésions péri-apicales ou des abcès dentaires pour 8/29 cas $(27 \%)$, des traitements dentaires pour $6 / 29$ cas (20\%), des caries dans $3 / 29$ cas (10\%), alors que 4/29 cas (10\%) étaient inconnus et 2/29 (7\%) étaient non documentés. Dans aucun des cas il n'était précisé si le site de l'infection dentaire avait été identifié en préopératoire, ni depuis combien de temps le patient avait une parodontite ou une lésion péri-apicale (Table 1). Les infections trouvées étaient hématogènes, avec un délai moyen de 32.9 mois entre l'insertion de la prothèse et l'apparition des premiers signes de l'infection (intervalle, 3 à 111 mois). Dans les études cas témoins, les cas étaient définis comme les patients avec signes cliniques d'infection de prothèses articulaires et les témoins étaient les patients sans signe d'infection de prothèses articulaires (Table 2). Seulement une étude cas témoins a signalé un examen dentaire préopératoire. Pour toutes les études cas-témoins, l'infection de la prothèse était associée à un abcès dentaire dans 6/224 cas (2.9\%). Dans les études de cohortes, l'exposition est définie comme "tout bilan ou traitement dentaire effectué avant la chirurgie" (Table 3). Les évaluations dentaires concernaient principalement la parodontite et les abcès dentaires. Seulement 4 études de cohortes ont fourni l'information de l'exposition. Selon les données des 12 études de cohortes, les traitements dentaires effectués avant la chirurgie étaient des avulsions dans 49/205 cas (24\%), des détartrages-surfaçages dans 78/205 cas (38\%), des soins conservateurs dans 37/205 cas (18\%), des couronnes dans $6 / 205$ cas (2.9\%), 
et des curetages de parodontites apicales et des traitements endodontiques dans 2/205 cas $(0.9 \%)$.

\subsection{Présence de facteurs de risques et comorbidités}

Dans les séries de cas, les patients qui avaient au moins une comorbidité associée représentent 17/29 (59\%) (Table 1). Dans les études cas témoins, les facteurs de risque associées à l'infection de prothèses articulaires étaient l'immunodéficience, la malnutrition, l'obésité, le diabète, le tabac et l'alcool, l'insuffisance rénale, la présence d'infections à distance, et l'antécédent de chirurgie sur le site. En ce qui concerne l'acte chirurgical, la durée de l'intervention est considérée comme un facteur de risque. Pour les pathologies articulaires, plus de cas de polyarthrite rhumatoïde étaient retrouvés chez les patients infectés. Dans les études de cohortes, les facteurs de risque les plus retrouvés étaient l'antécédent d'intervention chirurgicale sur le site, la polyarthrite rhumatoïde, le diabète, l'alcool, l'obésité et la présence de comorbidités associées.

\subsection{Evaluation qualitative}

Les tables 4 et 5 montrent les résultats de l'étude de l'évaluation qualitative. L'évaluation qualitative a été réalisée selon l'objectif de la revue systématique, et ne reflète pas la validité interne de chaque étude spécifique. Globalement, la qualité de nos études est faible. Parmi les études inclues, une présente un score NOS à 5, quatre ont un score NOS à 4, 10 ont un score NOS à 3 et une a un score NOS à 2. Aucune étude, dont l'objectif principal est d'évaluer l'association entre le bilan dentaire préopératoire et la survenue d'infection de prothèses articulaires, n'a été trouvée.

\section{Discussion}

La littérature a été examinée pour évaluer la pertinence d'un examen dentaire avant une chirurgie orthopédique. Cependant, en pratique, étant donné que cet examen dentaire est 
demandé, nous pensons qu'il est important de montrer la difficulté de mettre en évidence des arguments scientifiques pour justifier la réalisation de cet examen. A notre connaissance, cette étude est la première revue sur ce sujet.

En ce qui concerne les limites de notre travail, elles découlent du fait que nous n'avons trouvé aucune étude initialement faite pour répondre à la question "Existe-t-il un lien prouvé entre une infection orthopédique et la présence de pathologies dentaires en préopératoire?”. En fait, la plupart des études inclues traitent des questions connexes ("Les bactéries responsables d'infections orthopédiques sont-elles originaires de la cavité orale ?", Existe-t-il une association entre infections orthopédiques et santé bucco-dentaire avant ou après la chirurgie orthopédique?" ou "Quel est le délai entre la découverte d'un problème dentaire et la survenue d'une infection orthopédique?"). La réalisation de la revue de la littérature a été rendue complexe en raison de la grande hétérogénéité des études inclues. Les résultats initiaux ne permettent pas de tirer des conclusions définitives sur la relation entre l'évaluation dentaire préopératoire et les infections articulaires. Pour toutes les études, l'évaluation dentaire préopératoire n'est généralement pas renseignée et, si elle l'est, recense principalement des parodontites et des abcès dentaires. Bien que les études de cohortes révèlent une proportion légèrement inférieure d'infections articulaires chez les patients où l'évaluation dentaire préopératoire est réalisée, ce résultat est difficilement exploitable car peu d'études renseignent sur la réalisation de cet examen. Considérant l'hétérogénéité des études, le faible nombre de patients et le manque d'informations sur la réalisation de l'examen dentaire préopératoire, nous ne pouvons pas affirmer si ce bilan préopératoire est nécessaire ou non. Pour répondre à la question de la nécessité d'une évaluation préopératoire, plus de données démographiques sont requises pour comparer les patients dont l'intervention est programmée et qui vont réaliser le bilan préopératoire, et ceux traités en urgence dont le bilan n'a donc pas pu être réalisé. Lampley et al. [54] rapportent un bon exemple et suggèrent que 
le bilan dentaire préopératoire devrait être réévalué. Une étude sur l'impact économique qu'aurait la réalisation systématique du bilan dentaire serait également intéressante.

Cette revue rapporte que les patients présentant plus d'une comorbidité associée sont plus à risque de développer une infection de prothèses orthopédiques [60]. Tokarski et al. [61] montrent que l'examen dentaire est plus à risque de révéler des infections qui contre indiquent la pose de prothèses articulaires chez les patients avec facteurs de risque que chez ceux qui n'en ont pas. Cependant, il manque à cette étude le recul sur l'incidence des infections, la période de suivi s'arrêtant au moment de la pose de la prothèse. Cette étude montre une relation entre qualité de la santé bucco-dentaire et qualité de vie. De ce fait, il est important de réduire le nombre de facteurs de risque, un prérequis qui inclut la nécessité d'avoir une bouche saine [61]. De nombreuses études ont suggéré un lien entre diabète et maladies parodontales ou entre mauvaise hygiène dentaire et maladies cardiovasculaires [62]. Il est important de rétablir une bonne qualité de l'environnement buccal afin d'améliorer la qualité de vie et, ainsi, diminuer les facteurs de risque de développer une infection péri-prothétique. Bien que il n'y ait aucune preuve formelle pour ou contre l'évaluation dentaire préopératoire, il est conseillé de la réaliser dans le but de maintenir une hygiène dentaire favorable et ainsi réduire les facteurs de risque.

Dans le but de guider le chirurgien-dentiste et de mener à bien le bilan dentaire préopératoire, nous pouvons nous reposer sur les recommandations de prévention des maladies cardiaques. Les recommandations récentes autorisent plus de soins conservateurs, en fonction des facteurs de risque du patient et du délai entre l'examen dentaire et la chirurgie [63]. Pour les chirurgies urgentes (1 semaine), l'élimination des foyers infectieux actifs est recommandée. En revanche, pour les chirurgies programmées, les recommandations ont évolué et les chirurgiens-dentistes ont désormais un rôle préventif. Les foyers infectieux actifs doivent être éliminés mais il faut considérer les traitements alternatifs, comme les traitements 
parodontaux non chirurgicaux, les soins conservateurs et les traitements endodontiques pour les dents avec parodontite apicale non symptomatique, pulpite ou nécrose pulpaire ou même le traitement de caries profondes sur les molaires [63]. L'aspect le plus important est que ces recommandations suggèrent une collaboration étroite entre dentistes, cardiologues et chirurgiens cardiaques pour évaluer la santé bucco-dentaire, déterminer le protocole de suivi et les traitements nécessaires pour les patients avec des pathologies cardiaques. En attendant d'autres études sur le bilan dentaire préopératoire, les chirurgiens-dentistes peuvent mettre en place les recommandations pour les maladies cardiaques $[63,64]$.

\section{Conclusion}

Malgré le manque de preuves directes sur le lien entre infections dentaires et infections articulaires, il est néanmoins utile de maintenir une bonne santé bucco-dentaire dans la population générale et de réduire la présence de facteurs de risque qui pourraient réduire la qualité de vie. Une perspective intéressante serait d'étudier l'incidence des infections périprothétiques dans un groupe où le bilan dentaire préopératoire est réalisé et de relever des informations sur le nombre de dents restantes et leur état et le délai entre ce bilan et la pose de la prothèse.

Remerciements: Les auteurs remercient Susan Becker pour la relecture en langue anglaise.

Conflit d'intérêts: Dr. Reina rapporte des frais personnels de BBraun, frais personnels de Stryker, frais personnels de Zimmer, en dehors du travail soumis et Dr. Vergnes rapporte des frais personnels de BBraun, frais personnels de Sanofi Aventis France, Lilly France SAS et 
Pfizer SAS, en dehors du travail soumis. Les autres auteurs déclarent n'avoir aucun conflit d'intérêt.

Source de financement : aucune

\section{Contribution des auteurs :}

S Barrere, N Reina, D Maret: redaction, relecture ; O A Peters, L Rapp, JN Vergnes:

relecture. 


\section{Références}

1. Reina N, Delaunay C, Chiron P, Ramdane N, Hamadouche M. Infection as a cause of primary total hip arthroplasty revision and its predictive factors. Orthop Traumatol Surg Res 2013;99:555-61.

2. Jämsen E, Huhtala H, Puolakka T, Moilanen T. Risk factors for infection after knee arthroplasty. A register-based analysis of 43,149 cases. J Bone Joint Surg Am 2009;91:38-47.

3. Peersman G, Laskin R, Davis J, Peterson M. Infection in total knee replacement: a retrospective review of 6489 total knee replacements. Clin Orthop Relat Res 2001;1523.

4. Pulido L, Ghanem E, Joshi A, Purtill JJ, Parvizi J. Periprosthetic joint infection: the incidence, timing, and predisposing factors. Clin Orthop Relat Res 2008;466:1710-5.

5. Choong PFM, Dowsey MM, Carr D, Daffy J, Stanley P. Risk factors associated with acute hip prosthetic joint infections and outcome of treatment with a rifampin based regimen. Acta Orthop 2007;78:755-65.

6. Phillips JE, Crane TP, Noy M, Elliott TSJ, Grimer RJ. The incidence of deep prosthetic infections in a specialist orthopaedic hospital: a 15-year prospective survey. J Bone Joint Surg Br 2007;88:943-8.

7. Kurtz SM, Lau E, Schmier J, Ong KL, Zhao K, Parvizi J. Infection burden for hip and knee arthroplasty in the United States. J Arthroplasty 2008;23:984-91. 
8. Kurtz SM, Lau E, Ong K, Zhao K, Kelly M, Bozic KJ. Future young patient demand for primary and revision joint replacement: national projections from 2010 to 2030 . Clin Orthop Relat Res 2009;467:2606-12.

9. Triantafyllopoulos G, Stundner O, Memtsoudis S, Poultsides LA. Patient, surgery, and hospital related risk factors for surgical site infections following total hip arthroplasty. ScientificWorld Journal. 2015:979560.

10. Zhu Y, Zhang F, Chen W, Liu S, Zhang Q, Zhang Y. Risk factors for periprosthetic joint infection after total joint arthroplasty: a systematic review and meta-analysis. J Hosp Infect 2015;89:82-9.

11. Kunutsor SK, Whitehouse MR, Blom AW, Beswick AD, INFORM Team. PatientRelated Risk Factors for Periprosthetic Joint Infection after Total Joint Arthroplasty: A Systematic Review and Meta-Analysis. PloS One 2016;11:e0150866.

12. Del Pozo JL, Patel R. Clinical practice. Infection associated with prosthetic joints. N Engl J Med 2009; 361:787-94.

13. Murdoch DR, Roberts SA, Fowler Jr VG, Shah MA, Taylor SL, Morris AJ, et al. Infection of orthopedic prostheses after Staphylococcus aureus bacteremia. Clin Infect Dis 2001;32:647-9.

14. Berbari EF, Hanssen AD, Duffy MC, Steckelberg JM, Ilstrup DM, Harmsen WS, et al. Risk factors for prosthetic joint infection: case-control study. Clin Infect Dis $1998 ; 27: 1247-54$. 
15. Bongartz T, Halligan CS, Osmon DR, Reinalda MS, Bamlet WR, Crowson CS, et al. Incidence and risk factors of prosthetic joint infection after total hip or knee replacement in patients with rheumatoid arthritis. Arthritis Rheum 2008;59:1713-20.

16. Dowsey MM, Choong PFM. Obesity is a major risk factor for prosthetic infection after primary hip arthroplasty. Clin Orthop Relat Res 2008;466:153-8.

17. Marculescu CE, Mabry T, Berbari EF. Prevention of Surgical Site Infections in Joint Replacement Surgery. Surg Infect 2016;17:152-7.

18. Berbari EF, Osmon DR, Duffy MCT, Harmssen RNW, Mandrekar JN, Hanssen AD, et al. Outcome of prosthetic joint infection in patients with rheumatoid arthritis: the impact of medical and surgical therapy in 200 episodes. Clin Infect Dis 2006;42:216-23.

19. Trampuz A, Piper KE, Jacobson MJ, Hanssen AD, Unni KK, Osmon DR, et al. Sonication of removed hip and knee prostheses for diagnosis of infection. N Engl J Med 2007;357:654-63.

20. Ching DW, Gould IM, Rennie JA, Gibson PH. Prevention of late haematogenous infection in major prosthetic joints. J Antimicrob Chemother 1989;23:676-80.

21. Bartzokas CA, Johnson R, Jane M, Martin MV, Pearce PK, Saw Y. Relation between mouth and haematogenous infection in total joint replacements. BMJ 1994; 309:506-8.

22. Rubin R, Salvati EA, Lewis R. Infected total hip replacement after dental procedures. Oral Surg Oral Med Oral Pathol 1976; 41:18-23.

23. Gendron R, Grenier D, Maheu-Robert L. The oral cavity as a reservoir of bacterial pathogens for focal infections. Microbes Infect 2000;2:897-906. 
24. Vielpeau C, Lortat-Jacob A. Les prothèses totales de hanche infectées. Rev Chir Orthop 2002 ; 88(Suppl. 5) :162-216.

25. Dewhirst FE, Chen T, Izard J, Paster BJ, Tanner ACR, Yu W-H, et al. The human oral microbiome. J Bacteriol 2010;192:5002-17.

26. Lockhart PB, Brennan MT, Sasser HC, Fox PC, Paster BJ, Bahrani-Mougeot FK. Bacteremia associated with toothbrushing and dental extraction. Circulation 2008;117:3118-25.

27. Lockhart PB, Brennan MT, Thornhill M, Michalowicz BS, Noll J, Bahrani-Mougeot FK, et al. Poor oral hygiene as a risk factor for infective endocarditis-related bacteremia. $\mathrm{J}$ Am Dent Assoc 2009;140:1238-44.

28. Rademacher WMH, Walenkamp GHIM, Moojen DJF, Hendriks JGE, Goedendorp TA, Rozema FR. Antibiotic prophylaxis is not indicated prior to dental procedures for prevention of periprosthetic joint infections. Acta Orthop 2017;88:568-74.

29. Legout L, Beltrand E, Migaud H, Senneville E. Antibiotic prophylaxis to reduce the risk of joint implant contamination during dental surgery seems unnecessary. Orthop Traumatol Surg Res 2012;98:910-4.

30. American Dental Association Appointed Members of the Expert Writing and VotingPanels Contributing to the Development of American Academy of Orthopedic Surgeons Appropriate Use Criteria. American Dental Association guidance for utilizing appropriate use criteria in the management of the care of patients with orthopedic implants undergoing dental procedures. J Am Dent Assoc. 2017;148(2):57-59. 
31. Sollecito TP, Abt E, Lockhart PB, Truelove E, Paumier TM, Tracy SL, Tampi M, Beltrán-Aguilar ED, Frantsve-Hawley J. The use of prophylactic antibiotics prior to dental procedures in patients with prosthetic joints: Evidence-based clinical practice guideline for dental practitioners--a report of the American Dental Association Council on Scientific Affairs. J Am Dent Assoc. 2015;146(1):11-16.

32. Moher D, Liberati A, Tetzlaff J, Altman DG, PRISMA Group. Preferred reporting items for systematic reviews and meta-analyses: the PRISMA statement. J Clin Epidemiol 2009;62:1006-1.

33. Stang A. Critical evaluation of the Newcastle-Ottawa scale for the assessment of the quality of non randomized studies in meta-analyses. European Journal of Epidemiology, $2010 ; 25: 603-605$.

34. Sonohata M, Kitajima M, Kawano S, Mawatari M. Acute hematogenous infection of revision total hip arthroplasty by oral bacteria in a patient without a history of dental procedures: Case report. Open Orthop J 2014;8:56-9.

35. Bengtson S, Blomgren G, Knutson K, Wigren A, Lidgren L. Hematogenous infection after knee arthroplasty. Acta Orthop Scand 1987;58:529-34.

36. Lindqvist C, Slätis P. Dental bacteremia--a neglected cause of arthroplasty infections? Three hip cases. Acta Orthop Scand 1985;56:506-8.

37. Schurman DJ, Aptekar RG, Burton DS. Infection in total knee joint replacement, secondary to tooth abscess. West J Med 1976;125:226-7.

38. Cruess RL, Bickel WS, vonKessler KL. Infections in total hips secondary to a primary source elsewhere. Clin Orthop Relat Res 1975;106:99-101. 
39. Downes EM. Late infection after total hip replacement. J Bone Joint Surg Br $1977 ; 59: 42-4$.

40. Ahlberg A, Carlsson AS, Lindberg L. Hematogenous infection in total joint replacement. Clin Orthop Relat Res 1978;137:69-75.

41. Stinchfield FE, Bigliani LU, Neu HC, Goss TP, Foster CR. Late hematogenous infection of total joint replacement. J Bone Joint Surg Am 1980;62:1345-50.

42. Bauer T, Maman L, Matha C, Mamoudy P. Dental care and joint prostheses. Rev Chir Orthop 2007;93:607-18.

43. Kaar TK, Bogoch ER, Devlin HR. Acute metastatic infection of a revision total hip arthroplasty with oral bacteria after noninvasive dental treatment. J Arthroplasty $2000 ; 15: 675-8$.

44. Bartz H, Nonnenmacher C, Bollmann C, Kuhl M, Zimmermann S, Heeg K, et al. Micromonas (Peptostreptococcus) micros: unusual case of prosthetic joint infection associated with dental procedures. Int J Med Microbiol 2005;294:465-70.

45. Kessler B, Sendi P, Graber P, Knupp M, Zwicky L, Hintermann B, et al. Risk factors for periprosthetic ankle joint infection: a case-control study. J Bone Joint Surg Am 2012;94:1871-6.

46. Kaandorp CJ, Van Schaardenburg D, Krijnen P, Habbema JD, van de Laar MA. Risk factors for septic arthritis in patients with joint disease. A prospective study. Arthritis Rheum 1995;38:1819-25. 
47. Cordero-Ampuero J, de Dios M. What are the risk factors for infection in hemiarthroplasties and total hip arthroplasties? Clin Orthop Relat Res 2010;468:326877.

48. Andrews HJ, Arden GP, Hart GM, Owen JW. Deep infection after total hip replacement. J Bone Joint Surg Br 1981;63:53-7.

49. Aomori K, Kamada Y, Watanabe N, Fujiwara Y, Hosokawa M, Nakai M, et al. Dental examination prior to total joint arthroplasty as a means to prevent postoperative haematogenous infection. Jpn J Rheum Jt Surg 2003;22:223-8.

50. Barrington JW, Barrington TA. What is the true incidence of dental pathology in the total joint arthroplasty population? J Arthroplasty 2011;26:88-91.

51. Grogan TJ, Dorey F, Rollins J, Amstutz HC. Deep sepsis following total knee arthroplasty. Ten-year experience at the University of California at Los Angeles Medical Center. J Bone Joint Surg Am 1986;68:226-34.

52. Hamilton H, Jamieson J. Deep infection in total hip arthroplasty. Can J Surg J 2008;51:111-7.

53. Jacobsen PL, Murray W. Prophylactic coverage of dental patients with artificial joints: A retrospective analysis of thirty-three infections in hip prostheses. Oral Surg Oral Med Oral Pathol 1980;50:130-3.

54. Lampley A, Huang RC, Arnold WV, Parvizi J. Total joint arthroplasty: should patients have preoperative dental clearance? J Arthroplasty 2014;29:1087-90.

55. Maderazo EG, Judson S, Pasternak H. Late infections of total joint prostheses. A review and recommendations for prevention. Clin Orthop Relat Res 1988;229:131-42. 
56. Poss R, Thornhill TS, Ewald FC, Thomas WH, Batte NJ, Sledge CB. Factors influencing the incidence and outcome of infection following total joint arthroplasty. Clin Orthop Relat Res 1984;182:117-26.

57. Schmalzried TP, Amstutz HC, Au MK, Dorey FJ. Etiology of deep sepsis in total hip arthroplasty. The significance of hematogenous and recurrent infections. Clin Orthop Relat Res 1992;280:200-7.

58. Uçkay I, Lübbeke A, Emonet S, Tovmirzaeva L, Stern R, Ferry T, et al. Low incidence of haematogenous seeding to total hip and knee prostheses in patients with remote infections. J Infect 2009;59:337-45.

59. Waldman BJ, Mont MA, Hungerford DS. Total knee arthroplasty infections associated with dental procedures. Clin Orthop Relat Res 1997;343:164-72.

60. Guillain M, Tomeno B, Courpied JP, Commissionat Y, Boukhobza F, Al-Zriquat N. Complications infectieuses des prothèses articulaires et infection bucco-dentaire rapport à l'académie nationale de chirurgie dentaire, synthèse des données bibliographiques actuelles. Actual Odonto-Stomatol 2007;375-86.

61. Tokarski AT, Patel RG, Parvizi J, Deirmengian GK. Dental clearance prior to elective arthroplasty may not be needed for everyone. J Arthroplasty 2014;29:1729-32.

62. Loesche WJ. Association of the oral flora with important medical diseases. Curr Opin Periodontol 1997;4:21-8.

63. Millot S, Lesclous P, Colombier M-L, Radoi L, Messeca C, Ballanger M, et al. Position paper for the evaluation and management of oral status in patients with valvular disease: Groupe de Travail Valvulopathies de la Société Française de Cardiologie, Société 
Française de Chirurgie Orale, Société Française de Parodontologie et d’Implantologie Orale, Société Française d'Endodontie et Société de Pathologie Infectieuse de Langue Française. Arch Cardiovasc Dis 2017;110:482-94.

64. Cotti E, Arrica M, Di Lenarda A, Serri SB, Bassareo P, Padeletti L, Mercuro G. The perioperative dental screening and management of patients undergoing cardiothoracic, vascular surgery and other cardiovascular invasive procedures: A systematic review. Eur J Prev Cardiol 2017;24:409-425. 
Légende de la figure : Flowchart de la sélection de la littérature 
Table 1: Séries de cas

\begin{tabular}{|c|c|c|c|c|c|c|c|c|c|}
\hline $\begin{array}{l}\text { Auteur/ } \\
\text { Publication }\end{array}$ & $\begin{array}{l}\text { Nombres } \\
\text { de cas }\end{array}$ & $\begin{array}{l}\text { Age/ } \\
\text { sexe }\end{array}$ & $\begin{array}{l}\text { Comorbiditiés/ } \\
\text { Facteurs de } \\
\text { risques }\end{array}$ & Type et date de la prothèse & Antibioprophylaxie & $\begin{array}{l}\text { Examen dentaire } \\
\text { préopératoire }\end{array}$ & Délai d'apparition & Type d'infection & Foyers dentaires \\
\hline $\begin{array}{l}\text { Sonohata et } \\
\text { al. [34] }\end{array}$ & 1 & $53 / \mathrm{F}$ & $\begin{array}{l}\mathrm{RAS} \\
\mathrm{Nr}\end{array}$ & $\begin{array}{l}\text { Revision de PTH } \\
\mathrm{Nr}\end{array}$ & Cefazolin IV & $\mathrm{Nr}$ & 18 mois & $\begin{array}{l}\text { Hematogène } \\
\text { Str mutans gram+ }\end{array}$ & $\begin{array}{l}\text { caries } \\
\text { Str parasanguinus } \\
\text { (flore buccale) }\end{array}$ \\
\hline $\begin{array}{l}\text { Bengston et } \\
\text { al. [35] }\end{array}$ & $2^{*}$ & $\begin{array}{l}56 / F \\
73 / F\end{array}$ & $\begin{array}{l}\mathrm{Nr} \\
\mathrm{Nr}\end{array}$ & $\begin{array}{l}\text { PTG } \\
1973-1981\end{array}$ & $\mathrm{Nr}$ & $\mathrm{Nr}$ & $\begin{array}{l}18 \text { mois } \\
111 \text { mois }\end{array}$ & $\begin{array}{l}\text { Hematogène } \\
\text { Staph aureus }\end{array}$ & Inconnues \\
\hline $\begin{array}{l}\text { Lindqvist et } \\
\text { Slätis [36] }\end{array}$ & 3 & $\begin{array}{l}67 / \mathrm{H} \\
66 / \mathrm{F} \\
\\
84 / \mathrm{F} \\
\end{array}$ & $\begin{array}{l}\text { Arthrose } \\
\text { Post-traumatique } \\
\text { arthrose } \\
\text { Coxarthrose } \\
\text { bilateral } \\
\end{array}$ & $\begin{array}{l}\text { PTH1981 } \\
1982 \\
1978(\mathrm{~g})-1979(\mathrm{~d}) \\
\end{array}$ & $\begin{array}{l}\text { erythromycin }(-1 \mathrm{j} a ̀+3 \mathrm{j}) \\
\text { dicloxacillin } \\
\text { streptomycin }\end{array}$ & $\mathrm{Nr}$ & $\begin{array}{l}\text { ans } \\
2 \text { ans } \\
5 \text { ans } \\
\end{array}$ & $\begin{array}{l}\text { Hematogène } \\
\text { Str viridans }\end{array}$ & $\begin{array}{l}\text { Parodontite } \\
\text { parodontite } \\
\text { Parodontite + lésion péri-apicale }\end{array}$ \\
\hline $\begin{array}{l}\text { Schurman et } \\
\text { al. [37] }\end{array}$ & 1 & $61 / \mathrm{F}$ & $\begin{array}{l}\text { Arthrose } \\
\text { rhumatoide }\end{array}$ & $\begin{array}{l}\text { Prothèse de genou bilateral } \\
1973\end{array}$ & Cephalosporin IV (d4) & $\mathrm{Nr}$ & 2 ans & $\begin{array}{l}\text { Hematogène } \\
\text { Cocci gram+ } \\
\text { Staphaureus } \\
\end{array}$ & Abcès incisive mandibulaire droite \\
\hline $\begin{array}{l}\text { Cruess et al. } \\
{[38]}\end{array}$ & $2^{*}$ & $\begin{array}{l}72 / F \\
64 / F\end{array}$ & $\begin{array}{l}\text { RAS } \\
\text { Arthrose } \\
\text { dégénérative }\end{array}$ & $\begin{array}{l}\text { PTH } \\
1970\end{array}$ & $\begin{array}{l}\mathrm{Nr} \\
\text { Ampicillin + dimethoxyphenyl penicillin } \\
\text { (preop d5) }\end{array}$ & $\mathrm{Nr}$ & $\begin{array}{l}2 \text { ans } \\
3 \text { mois }\end{array}$ & $\begin{array}{l}\text { Hematogène } \\
\text { Staph epidermidis } \\
\text { non groupe A Str }\end{array}$ & $\begin{array}{l}\text { Molaire infectée } \\
\mathrm{Nr}\end{array}$ \\
\hline Downes [39] & $3^{*}$ & $\begin{array}{l}69 / \mathrm{F} \\
54 / \mathrm{F} \\
62 / \mathrm{H}\end{array}$ & $\begin{array}{l}\text { Ostéoarthrose } \\
\mathrm{Nr} \\
\text { Osteo-arthrose }\end{array}$ & $\begin{array}{l}\text { PTH } \\
1968(1)-1969(\mathrm{r}) \\
1969 \\
1971\end{array}$ & $\mathrm{Nr}$ & $\mathrm{Nr}$ & $\begin{array}{l}1-2 \text { ans } \\
5 \text { ans } \\
3 \text { ans }\end{array}$ & $\begin{array}{l}\text { Hematogène } \\
\text { Staph aureus } \\
\text { Str } \beta \text { hemolytic }\end{array}$ & $\begin{array}{l}\text { Abcès parotide } \\
\mathrm{Nr} \\
\text { parodontite }\end{array}$ \\
\hline $\begin{array}{l}\text { Ahlberg et al. } \\
\text { [40] }\end{array}$ & $3^{*}$ & $\begin{array}{l}76 / \mathrm{F} \\
70 / \mathrm{F} \\
39 / \mathrm{H}\end{array}$ & $\begin{array}{l}\text { Ostéoarthrose } \\
\text { Arthrose } \\
\text { rhumatoïde } \\
\text { spondylarthrite } \\
\end{array}$ & $\begin{array}{l}\text { PTH(x2) PTG } \\
1970(\mathrm{~g})-1971(\mathrm{~d}) \\
1975 \\
1973-74 \\
\end{array}$ & $\begin{array}{l}\text { Cloxacillin } \\
\text { Cloxacillin (2semaines) } \\
\mathrm{Nr}\end{array}$ & $\mathrm{Nr}$ & $\begin{array}{l}3 \text { ans } \\
1 \text { an } \\
3 \text { ans }\end{array}$ & $\begin{array}{l}\text { Hematogène } \\
\text { Pneumococcal } \\
\text { Str } \beta \text { hemolytic } \\
\text { Staph aureus } \\
\end{array}$ & Unknown sources \\
\hline $\begin{array}{l}\text { Stinchfield et } \\
\text { al. [41] }\end{array}$ & $2^{*}$ & $\begin{array}{l}60 / \mathrm{H} \\
56 / \mathrm{F}\end{array}$ & $\begin{array}{l}\text { Arthrose } \\
\text { rhumatoïde } \\
\text { Ostéoarthrose }\end{array}$ & $\begin{array}{l}\text { PTH-PTG } \\
1975 \\
1976\end{array}$ & Ampicillin + oxacillin & $\begin{array}{l}\text { Examen médical } \\
\text { complet }\end{array}$ & $\begin{array}{l}5 \text { mois } \\
1 \text { an }\end{array}$ & $\begin{array}{l}\text { Hematogène } \\
\text { Str group G } \\
\text { Staph aureus }\end{array}$ & $\begin{array}{l}\text { Abcès dentaire } \\
\text { Caries dentaires }\end{array}$ \\
\hline $\begin{array}{l}\text { Bartzokas et } \\
\text { al. [21] }\end{array}$ & 4 & $\begin{array}{l}83 / \mathrm{Nr} \\
58 / \mathrm{Nr} \\
64 / \mathrm{Nr} \\
75 / \mathrm{Nr}\end{array}$ & $\begin{array}{l}\text { Ostéoarthrose } \\
\text { Ostéoarthrose } \\
\text { Ostéoarthrose } \\
\text { Antécédent de } \\
\text { douleur sur PTH } \\
\end{array}$ & $\begin{array}{l}\text { Prothèse de genou } \\
\mathrm{Nr}\end{array}$ & $\mathrm{Nr}$ & $\mathrm{Nr}$ & $\mathrm{Nr}$ & $\begin{array}{l}\text { Hematogène } \\
\text { Str sangunis }\end{array}$ & $\begin{array}{l}\text { Parodontite }+ \text { infection péri-apicale } \\
\text { Augmentation poche parodontale }\end{array}$ \\
\hline $\begin{array}{l}\text { Bauer et al. } \\
\text { [42] }\end{array}$ & 6 & $\mathrm{Nr}$ & Aucun & $\begin{array}{l}\text { PTH(x4)-TPTGx2) } \\
\mathrm{Nr}\end{array}$ & $\mathrm{Nr}$ & $\mathrm{Nr}$ & $\begin{array}{l}24 \text { mois } \\
84 \text { mois } \\
18 \text { mois } \\
96 \text { mois } \\
4 \text { mois } \\
72 \text { mois }\end{array}$ & $\begin{array}{l}\text { Hematogène } \\
\text { Str intermedius } \\
\text { Str mitis } \\
\text { Str adjacent } \\
\text { Abiotrophia } \\
\text { Str } \beta \text { hemolytic }\end{array}$ & $\begin{array}{l}\text { Extractions } \\
\text { carie } \\
\text { soins } \\
\text { Abcès } \\
\text { Extraction } \\
\text { soins }\end{array}$ \\
\hline $\begin{array}{l}\text { Kaar et } \\
\text { al. }[43]\end{array}$ & 1 & $67 / \mathrm{H}$ & $\mathrm{Nr}$ & $\begin{array}{l}\text { PTH revision } \\
\mathrm{Nr}\end{array}$ & cefazolin & $\mathrm{Nr}$ & $11 \mathrm{mois}$ & $\begin{array}{l}\text { Hematogène } \\
\text { Str intermedius } \\
\text { Str mutans }\end{array}$ & Détartrage Supra gingival \\
\hline $\begin{array}{l}\text { Bartz et al. } \\
\text { [44] }\end{array}$ & 1 & $63 / \mathrm{F}$ & $\begin{array}{l}\text { Arthrose de } \\
\text { Lyme }\end{array}$ & $\begin{array}{l}\mathrm{PTH} \\
\mathrm{Nr}\end{array}$ & $\mathrm{Nr}$ & $\mathrm{Nr}$ & 9 ans & $\begin{array}{l}\text { Hematogène } \\
\text { Peptostreptococcus } \\
\text { micros }\end{array}$ & $\begin{array}{l}\text { Parodontite } \\
\text { Infection Peri apical } \\
\text { Molaire infectée }\end{array}$ \\
\hline
\end{tabular}

*seulement les cas de sources dentaires inconnues Nr: non renseigné, H homme, F: femme, PTH: prothèse totale de hanche,PTG: prothèse totale de genou, str: streptococcus, staph:

Staphylococcus 
Table 2: Etude Cas Témoins

\begin{tabular}{|c|c|c|c|c|c|c|c|c|c|}
\hline $\begin{array}{l}\text { Auteur/ } \\
\text { publication }\end{array}$ & $\begin{array}{l}\text { Non } \\
\text { et d }\end{array}$ & $\begin{array}{l}\text { nbre de cas } \\
\text { témoins }\end{array}$ & $\begin{array}{l}\text { Age/ } \\
\text { sexe }\end{array}$ & Pathologie articulaire & Comorbidités & $\begin{array}{l}\text { Examen } \\
\text { dentaire } \\
\text { préopératoire }\end{array}$ & Type d'infection & Délai d'apparition & Foyers infectieux dentaires \\
\hline \multirow[t]{2}{*}{$\begin{array}{l}\text { Peersman } \\
\text { et al. [3] }\end{array}$} & שే & $\begin{array}{l}116 \\
\text { prothèses } \\
\text { (3 exclus) } \\
\text { (114 } \\
\text { patients : } \\
1 \text { exclus) }\end{array}$ & $\begin{array}{l}\mathrm{Nr} \\
1 \text { cas } \\
\text { pour } 2 \\
\text { témoins }\end{array}$ & $\begin{array}{l}62 \% \text { : ostéoarthroses } \\
20 \% \text { : Arthroses rhumatoïdes } \\
11 \% \text { : ostéoarthroses post } \\
\text { traumatiques } \\
7 \% \text { autres }\end{array}$ & $\begin{array}{l}96 \% \geq 1 \text { comorbiditiés } \\
\text { Comorbiditiés augmentant le risque } \\
\text { d'infection: } \\
\text { Antécédent de première chirurgie } \\
\text { Thérapie Immunosuppressive } \\
\text { Hypokaliémie } \\
\text { Malnutrition } \\
\text { Diverticule } \\
\text { Infection éloignée } \\
\text { Diabète sucré } \\
\text { Obésité } \\
\text { Tabac } \\
\text { Problèmes rénaux } \\
\text { Hypothyroödie } \\
\text { Alcoolisme } \\
\text { Durée chirurgie }\end{array}$ & $\mathrm{OK}$ & $\begin{array}{l}14 \% \text { ISO } \\
86 \% \text { infection profonde } \\
34,5 \% \text { hématogènes (=56.5\% infections profondes } \\
\text { tardives) } \\
35 \% \text { Staph aureus } \\
15 \% \text { Staph epidermidis } \\
6 \% \text { Str group B } \\
4 \% \text { Escherichia coli, Staph aureus } \mathrm{Mr} \\
12 \% \text { autres } \\
9 \% \text { poly microbiennes } \\
19 \% \text { inconnus }\end{array}$ & $\begin{array}{l}<3 \text { mois } \\
29 \%<3 \text { mois } \\
71 \%>3 \text { mois }\end{array}$ & $\begin{array}{l}2.6 \% \\
\text { Abcès dentaire }\end{array}$ \\
\hline & 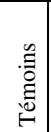 & $\begin{array}{l}236 \\
\text { patients }\end{array}$ & $\mathrm{Nr}$ & $\mathrm{Nr}$ & $\begin{array}{l}80 \% \geq 1 \text { comorbiditiés } \\
\text { Différence significative du nombre de } \\
\text { comorbidités }\end{array}$ & OK & & & \\
\hline \multirow[t]{3}{*}{$\begin{array}{l}\text { Kessler et } \\
\text { al. [45] }\end{array}$} & שี & $\begin{array}{l}26 \\
\text { patients }\end{array}$ & $\begin{array}{l}\sigma 61.4 \\
\text { ans } \\
39 \% \mathrm{H} \\
61 \% \mathrm{~F}\end{array}$ & $\begin{array}{l}31 \% \text { ostéoarthroses } \\
8 \% \text { Arthroses rhumatoïdes } \\
54 \% \text { ostéoarthroses traumatique } \\
15 \% \text { révision }\end{array}$ & $\begin{array}{l}\text { IMC moyen } 26,7 \mathrm{~kg} / \mathrm{m}^{2} \\
7.7 \% \text { diabète sucré } \\
11.5 \% \text { corticotheŕrapies } \\
3.8 \% \text { problèmes rénaux chroniques } \\
3.8 \% \text { cardiopathies chroniques } \\
3.8 \% \text { problèmes dermatologiques } \\
\text { chroniques } \\
3.8 \% \text { polyneuropathie } \\
3.8 \% \text { occlusion artérielle } \\
19 \% \text { tabac }\end{array}$ & $\mathrm{Nr}$ & $\begin{array}{l}\text { 85\% exogènes } \\
15 \% \text { hématogènes } \\
35 \% \text { Staph aureus (lepisode du aux Mr) } \\
31 \% \text { Staph. coagulase - } \\
15 \% \text { Enterococcus } \\
12 \% \text { Enterobacter } \\
8 \% \text { Klebsiella pneumoniae. Propionibacterium acnes } \\
4 \% \text { Str milleri, Pseudomonas aeruginosa, } \\
\text { Achromobacter spp } \\
15 \% \text { poly microbiennes }\end{array}$ & $\begin{array}{l}\text { Moyenne } 193 \text { jours } \\
(10-376 \text { jours }) \\
23 \%<90 \text { jours } \\
62 \% 90 \text { jours-2ans } \\
15 \%>2 \text { ans }\end{array}$ & $3.8 \%$ abcès dentaire (1cas) \\
\hline & 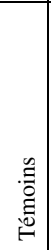 & $\begin{array}{c}52 \\
\text { patients }\end{array}$ & $\begin{array}{c}64 \\
\text { ans } \\
39 \% \mathrm{H} \\
61 \% \mathrm{~F}\end{array}$ & $\begin{array}{l}31 \% \text { ostéoarthroses } \\
6 \% \text { Arthroses rhumatoides } \\
64 \% \text { ostéoarthroses } \\
\text { traumatiques } \\
\\
48 \% \text { ostéoarthroses }\end{array}$ & $\begin{array}{l}\text { IMC moyen } 26,5 \mathrm{~kg} / \mathrm{m}^{2} \\
1.9 \% \text { diabète sucré } \\
5.7 \% \text { tumeurs malignes } \\
5.7 \% \text { corticothérapies } \\
5.7 \% \text { cardiopathies chroniques } \\
1.9 \% \text { problèmes dermatologiques } \\
\text { chroniques } \\
1.9 \% \text { polyneuropathie } \\
10 \% \text { tabac }\end{array}$ & $\mathrm{Nr}$ & & & \\
\hline & & $\begin{array}{l}52 \\
\text { patients }\end{array}$ & $\begin{array}{c}\sim 64.6 \\
\text { ans } \\
56 \% \mathrm{H} \\
34 \% \mathrm{~F} \\
\end{array}$ & $\begin{array}{l}2 \% \text { Arthroses rhumatoïdes } \\
50 \% \text { ostéoarthroses } \\
\text { traumatiques } \\
2 \% \text { révision }\end{array}$ & $\begin{array}{l}\text { IMC moyen } 27.5 \mathrm{~kg} / \mathrm{m}^{2} \\
9.6 \% \text { diabète sucré } \\
5.7 \% \text { cardiopathies chroniques } \\
3.8 \% \text { occlusion artérielle } \\
21 \% \text { tabac }\end{array}$ & $\mathrm{Nr}$ & & & \\
\hline $\begin{array}{l}\text { Kaandorp } \\
\text { et al. [46] }\end{array}$ & లే & $\begin{array}{l}37 \\
\text { patients }\end{array}$ & $\begin{array}{l}\sim 65 \\
\text { ans } \\
38 \% \mathrm{H} \\
62 \% \mathrm{~F}\end{array}$ & $\begin{array}{l}67 \% \text { Arthroses rhumatoïdes } \\
5 \% \text { ostéo-arthroses sur un autre } \\
\text { site } \\
3 \% \text { spondylarthrose } \\
\text { ankylosante } \\
14 \% \text { ACJ, lupus érythémateux } \\
19 \% \text { ostéoarthroses hanche ou } \\
\text { genou }\end{array}$ & $\begin{array}{l}5 \% \text { malignité } \\
11 \% \text { diabètes } \\
3 \% \text { pathologie du foie } \\
32 \% \text { thérapie immunosuppressive } \\
86 \% \text { infection (cutanée, pulmonaire, } \\
\text { urinaire) }\end{array}$ & $\mathrm{Nr}$ & $\begin{array}{l}41 \% \text { infection du site chirurgical } \\
59 \% \text { hématogènes } \\
40 \% \text { Staph aureus }\end{array}$ & $\mathrm{Nr}$ & $\mathrm{Nr}$ \\
\hline
\end{tabular}




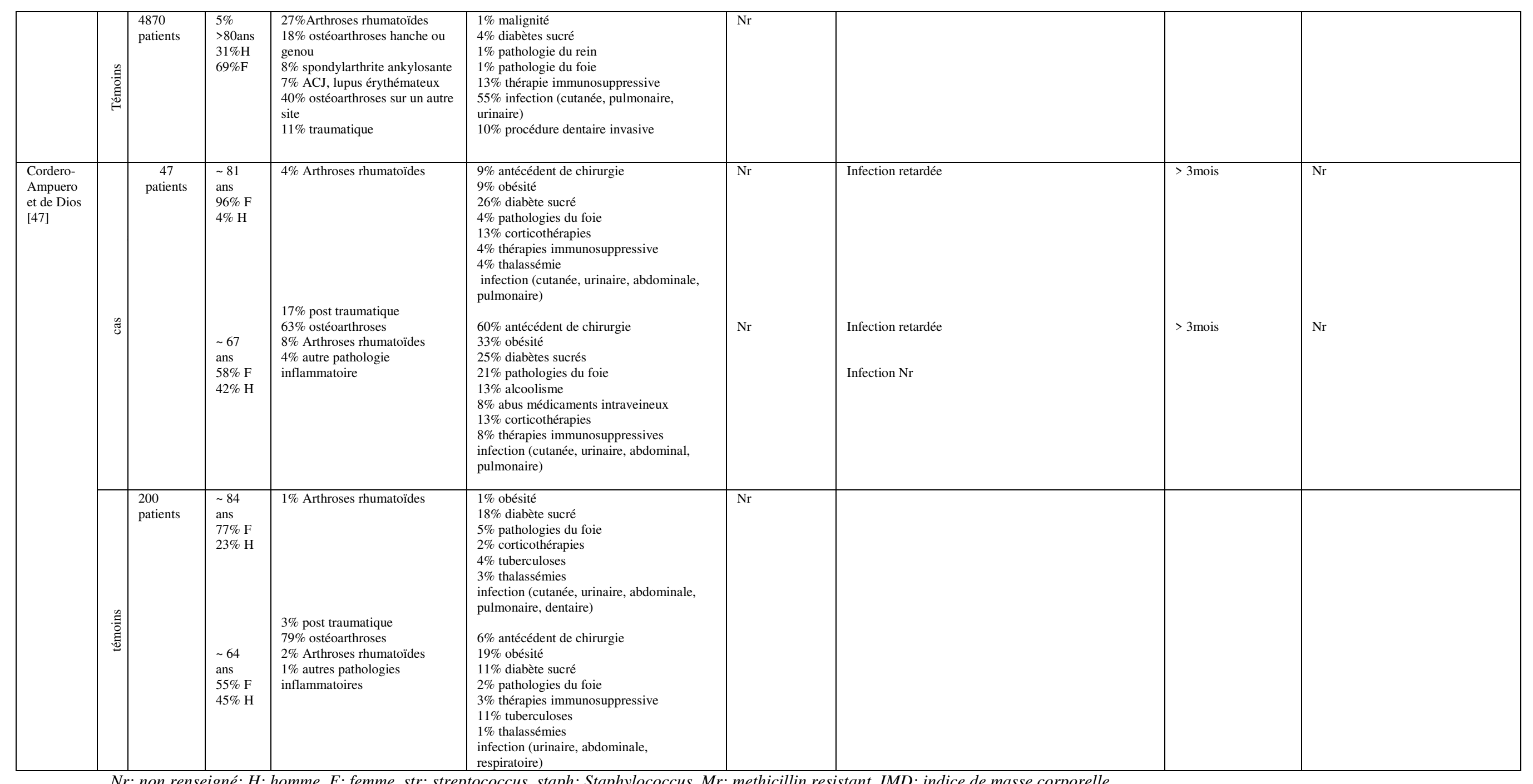

Nr: non renseigné; H: homme, F: femme, str: streptococcus, staph: Staphylococcus, Mr: methicillin resistant, IMD: indice de masse corporelle

Table 3: Etudes de Cohorte

\begin{tabular}{|c|c|c|c|c|c|c|c|c|c|}
\hline $\begin{array}{l}\text { Auteurs/ } \\
\text { Publication }\end{array}$ & $\begin{array}{l}\text { Nombre de } \\
\text { cas }\end{array}$ & $\begin{array}{l}\text { Moyenne d'age } \\
\text { et sexe }\end{array}$ & $\begin{array}{l}\text { Type de prothèse } \\
\text { et pathologie } \\
\text { articulaire }\end{array}$ & $\begin{array}{l}\text { Comorbiditiés et } \\
\text { facteurs de risques }\end{array}$ & $\begin{array}{l}\text { Examen } \\
\text { dentaire }\end{array}$ & $\begin{array}{l}\text { Traitements dentaires } \\
\text { avant chirurgie } \\
\text { orthopédique }\end{array}$ & Incidence/ Type infection & Délai d'apparition & Foyers dentaires \\
\hline $\begin{array}{l}\text { Andrews et } \\
\text { al. [48] }\end{array}$ & $\begin{array}{l}\text { 64 PTH } \\
\text { (65 patients }\end{array}$ & $\begin{array}{l}\mathrm{Nr} \\
81.5 \% \mathrm{~F}\end{array}$ & $\begin{array}{l}\text { PTH } \\
1.8 \%\end{array}$ & $\begin{array}{l}\text { Précédente } \\
\text { intervention }\end{array}$ & $\mathrm{Nr}$ & $\mathrm{Nr}$ & $\begin{array}{l}\text { Incidence } 3.9 \% \\
\text { Infection précoce }\end{array}$ & $\begin{array}{l}54 \%<4 \text { mois } \\
18 \%<1 \text { an }\end{array}$ & $\mathrm{Nr}$ \\
\hline
\end{tabular}




\begin{tabular}{|c|c|c|c|c|c|c|c|c|c|}
\hline & $\begin{array}{l}\text { infectés : } 4 \\
\text { morts) }\end{array}$ & $18.5 \% \mathrm{H}$ & $\begin{array}{l}\text { ostéoarthroses } \\
10.5 \% \text { Arthroses } \\
\text { rhumatoïdes } \\
7.2 \% \text { Révision } \\
7.7 \% \\
\text { spondylarthrite } \\
\text { ankylosante } \\
20 \% \text { traumatisme }\end{array}$ & $\begin{array}{l}\text { Arthroses } \\
\text { rhumatoïdes } \\
\text { Anticoagulant } \\
\text { ISO }\end{array}$ & & & $\begin{array}{l}\text { Infection tardive } \\
17.6 \% \text { infection } \\
\text { hématogène } \\
32 \% \text { Staph aureus } \\
15 \% \text { Staph. albus } \\
15 \% \text { Str } \\
11 \% \text { Escherichia coli } \\
8 \% \text { Proteus spp } \\
9 \% \\
\text { Klebsiella/Enterobacter/S } \\
\text { erratia } \\
1 \% \text { Salmonella } \\
7 \% \text { Pseudomonas } \\
4 \% \text { anaérobie }\end{array}$ & $28 \%<10$ ans & \\
\hline $\begin{array}{l}\text { Aomori et al. } \\
{[49]}\end{array}$ & $\begin{array}{l}105 \text { patients } \\
\text { (113 } \\
\text { prothèses) }\end{array}$ & $\begin{array}{l}\text { 7ans } \\
(43-87 \text { ans }) \\
77.1 \% \mathrm{~F} \\
22.8 \% \mathrm{H}\end{array}$ & $\begin{array}{l}38 \% \text { PTH } \\
58.4 \% \text { PTG } \\
2.6 \% \text { PTC } \\
0.9 \% \text { PTE } \\
82.3 \% \\
\text { ostéoarthroses } \\
17.7 \% \text { Arthroses } \\
\text { rhumatoïdes }\end{array}$ & $\mathrm{Nr}$ & $\begin{array}{l}\begin{array}{l}\text { Panoramique } \\
\text { dentaire }\end{array} \\
10-48 \text { jours } \\
\text { (moyenne } 30 \mathrm{j} \text { ) } \\
\text { avant la pose } \\
74.3 \% \\
\text { Parodontites } \\
17 \% \text { édentés }\end{array}$ & $\begin{array}{l}22.1 \% \text { avulsions } \\
0.9 \% \text { curetage poche } \\
\text { parodontale } \\
73.4 \% \text { surfaçage } \\
4.4 \% \text { eviction carieuse }\end{array}$ & $\begin{array}{l}\text { Incidence } \\
1.9 \% \\
\text { Staph epidermidis }\end{array}$ & $\begin{array}{l}0.9 \% 1 \text { semaine } \\
0.9 \% \text { 10mois-1an }\end{array}$ & $\begin{array}{c}\text { Parodontite } \\
\text { Avulsion (2mois avant la chirurgie) } \\
\text { Pas de foyers actifs après la pose } \\
\text { Parodontite (pas de foyers actifs après la pose) }\end{array}$ \\
\hline $\begin{array}{l}\text { Barrington et } \\
\text { Barrington } \\
\text { [50] }\end{array}$ & 100 & $\begin{array}{l}64 \text { ans } \\
54 \% \mathrm{~F} \\
46 \% \mathrm{H}\end{array}$ & $\begin{array}{l}75 \% \text { première } \\
\text { chirurgie } \\
\text { (31\% PTH, 44\% } \\
\text { PTG) } \\
25 \% \text { révision } \\
\text { (15\% PTH, 10\% } \\
\text { PTG) } \\
\text { Nr }\end{array}$ & $\mathrm{Nr}$ & $\begin{array}{l}\text { OK } \\
23 \% \text { caries dont } \\
1 \% \text { de } \\
\text { parodontite }\end{array}$ & $\begin{array}{l}32 \text { évictions carieuse } \\
26 \text { avulsions (abcès) } \\
6 \text { couronnes } \\
1 \text { traitement } \\
\text { endodontique1 } \\
\text { détartrage/ surfaçage }\end{array}$ & $\begin{array}{l}0 \% \text { d'infection } \\
\text { Pas d'infection retrouvée }\end{array}$ & $<90$ jours & Aucun \\
\hline $\begin{array}{l}\text { Grogan et al. } \\
\text { [51] }\end{array}$ & $\begin{array}{l}12^{*} \\
\text { (13 } \\
\text { prothèses) }\end{array}$ & $\begin{array}{l}\mathrm{Nr} \\
41.6 \% \mathrm{H} \\
58.3 \% \mathrm{~F}\end{array}$ & $\begin{array}{l}\text { PTG } \\
84.6 \% \text { première } \\
15.4 \% \text { révision } \\
41.6 \% \text { Arthroses } \\
\text { rhumatoödes } \\
50 \% \\
\text { ostéoarthroses } \\
8.3 \% \text { lupus } \\
\text { érythémateux }\end{array}$ & $\begin{array}{l}\text { Révisions de } \\
\text { prothèse } \\
\text { Type de prothèses }\end{array}$ & $\mathrm{Nr}$ & $\mathrm{Nr}$ & $\begin{array}{l}\text { Incidence } 1.71 \% \\
50 \% \text { inconnus } \\
7.1 \% \text { ISO } \\
42.8 \% \text { hématogènes } \\
21.4 \% \text { Staph aureus, Str } \\
\text { micrococcus } \\
7.1 \% \text { Escherichia coli, } \\
\text { Proteus mirabilis, } \mathrm{P} \\
\text { maroxella, Str viridans, } \\
\text { Enterobacter aérogenes, } \\
\text { Staph epidermidis, Str } \\
\text { group A, listeria }\end{array}$ & $\begin{array}{l}2 \text { semaines- } 40 \text { mois } \\
\text { (moyenne } 8.3 \text { mois) } \\
\text { Infection hématogène } \\
3.5-40 \text { mois } \\
\text { (moyenne16.4 mois) }\end{array}$ & Pas d'antécédent d'infection orale \\
\hline $\begin{array}{l}\text { Hamilton et } \\
\text { Jamieson } \\
{[52]}\end{array}$ & $\begin{array}{l}1993 \\
\text { opérations }\end{array}$ & $\mathrm{Nr}$ & $\begin{array}{l}\text { PTH } \\
84 \% \text { première } \\
16 \% \text { révision }\end{array}$ & $\begin{array}{l}\text { Transfusion } \\
\text { sanguine } \\
\text { type d'anesthésie } \\
\text { Tumeur maligne } \\
\text { radio/ chimio } \\
\text { immunosuppresseurs } \\
\text { Arthroses } \\
\text { rhumatoödes } \\
\text { Diabètes sucrés } \\
\text { alcoolisme }\end{array}$ & $\mathrm{Nr}$ & $\mathrm{Nr}$ & $\begin{array}{l}2 \% \text { infections } \\
31 \% \text { Staph aureus } \\
21 \% \text { Staph epidermidis }\end{array}$ & $\mathrm{Nr}$ & $\mathrm{Nr}$ \\
\hline $\begin{array}{l}\text { Jacobsen et } \\
\text { Murray [53] }\end{array}$ & $\begin{array}{l}33 \text { (prothèses } \\
\text { infectées) }\end{array}$ & $\mathrm{Nr}$ & $\mathrm{Nr}$ & $\mathrm{Nr}$ & $\mathrm{Nr}$ & $\mathrm{Nr}$ & $\begin{array}{l}\text { Incidence }: 1.8 \% \\
\text { Infection précoce } \\
\text { Infection tardive } \\
43 \% \text { Staph aureus } \\
\text { (infections tardives ++) } \\
17 \% \text { Staph epidermidis } \\
17 \% \text { Pseudomonas }\end{array}$ & $\begin{array}{c}6 \text { mois } \\
\text { moyenne } 34 \text { mois } \\
(17-48)\end{array}$ & $\begin{array}{l}\text { 3\% associés à un foyer dentaire } \\
\text { Abcès préri-apical }\end{array}$ \\
\hline
\end{tabular}




\begin{tabular}{|c|c|c|c|c|c|c|c|c|c|}
\hline & & & & & & & $\begin{array}{l}\text { aeruginosa } \\
6 \% \text { Str. } \alpha \text { and } \\
\text { Enterococcus } \\
3 \% \text { Str. } \gamma \text { and } \beta, \\
\text { Peptostreptococcus, } \\
\text { Candida tropicalis. }\end{array}$ & & \\
\hline \multirow[t]{2}{*}{$\begin{array}{l}\text { Lampley et } \\
\text { al. [54] }\end{array}$} & $\begin{array}{l}365 \\
\text { (examens } \\
\text { dentaire) }\end{array}$ & $\begin{array}{l}62.4 \text { ans } \\
(16-88) \\
44 \% \mathrm{H} \\
56 \% \mathrm{~F}\end{array}$ & $\begin{array}{l}47 \% \text { PTG: } \\
-90 \% \text { première } \\
-9.4 \% \text { révision } \\
-0.6 \% \\
\text { arthroplasties } \\
\\
53 \% \text { PTH: } \\
-89 \% \text { première } \\
-11 \% \text { révision }\end{array}$ & $\begin{array}{l}\text { IMC } 30.6 \\
\text { Index de } \\
\text { comorbidité: } 2\end{array}$ & $\begin{array}{l}84 \% \mathrm{OK} \\
8.8 \% \\
\text { parodontites } \\
6.0 \% \text { édentés } \\
1.6 \% \text { pas vus }\end{array}$ & $\begin{array}{l}2 \% \text { non traités (pas de } \\
\text { chirurgie) } \\
6.8 \% \text { traités }\end{array}$ & $\begin{array}{l}\text { Incidence } 1.7 \% \\
50 \% \text { Staph aureus } \\
33 \% \text { Staph coagulase - } \\
16 \% \text { Pseudomonas } \\
\text { aeruginosa } \\
16 \% \text { Peptostreptococcus } \\
\text { magnus } \\
16 \% \text { Enterococcus faecalis } \\
16 \% \text { Str group B }\end{array}$ & $\begin{array}{l}<6 \text { mois } \\
\text { Moyenne } 68.5 \text { jours } \\
(36-166)\end{array}$ & $\begin{array}{l}1 \text { patient nécessitait un traitement avant la } \\
\text { chirurgie }\end{array}$ \\
\hline & 218 (sans) & $\begin{array}{l}78.7 \text { ans } \\
(42-101) \\
32 \% \mathrm{H} \\
68 \% \mathrm{~F} \\
\end{array}$ & $\begin{array}{l}\text { 63\%PTH } \\
37 \% \text { hemi PH } \\
\mathrm{Nr}\end{array}$ & $\begin{array}{l}\text { IMC 24 } \\
\text { Index de } \\
\text { comorbidité: } 3.7\end{array}$ & aucun & aucun & $\begin{array}{l}\text { Incidence } 2.5 \% \\
\text { Staph coagulase - }\end{array}$ & $\begin{array}{l}<1 \text { mois } \\
\text { Moyenne } 16 \text { jours } \\
(9-28)\end{array}$ & aucun \\
\hline $\begin{array}{l}\text { Maderazo et } \\
\text { al. [55] }\end{array}$ & $\begin{array}{l}24 \text { (nfections } \\
\text { tardives) }\end{array}$ & 56 ans $(27-86)$ & $\begin{array}{l}58 \% \text { PTH } \\
46 \% \text { PTG } \\
46 \% \\
\text { ostéoarthroses } \\
29 \% \text { Arthroses } \\
\text { rhumatoïdes } \\
21 \% \text { traumatisme } \\
4 \% \text { nécrose } \\
\text { vasculaire } \\
\text { (radiothérapie) } \\
4 \% \text { Arthroses } \\
\text { rhumatoïdes } \\
\text { juveniles } \\
\end{array}$ & $\mathrm{Nr}$ & $\mathrm{Nr}$ & $\mathrm{Nr}$ & $\begin{array}{l}\text { Incidence } 1.7 \% \\
42 \% \text { hématogènes. } \\
46 \% \text { ISO } \\
\text { propionibacterium } \\
\text { Staph. epidermidis } \\
\text { Staph aureus }\end{array}$ & 12 mois -6 ans & $\begin{array}{l}\text { Abcès péri-apical } \\
\text { Parodontites (avulsions) } \\
\text { avulsions }\end{array}$ \\
\hline $\begin{array}{l}\text { Poss et al. } \\
{[56]^{1}}\end{array}$ & $\begin{array}{l}4240 \\
\text { (prothèses) }\end{array}$ & $\begin{array}{l}65 \text { ans } \\
\text { (Ostéoarthrose) } \\
55 \text { ans } \\
\text { (Arthroses } \\
\text { rhumatoïdes) } \\
\mathrm{Nr}\end{array}$ & $\begin{array}{l}2012 \text { PTH } \\
1957 \text { PTG (metal- } \\
\text { plastic) } \\
156 \text { PTG (metal- } \\
\text { metal) } \\
115 \text { PTE } \\
90 \% \\
\text { ostéoarthroses + } \\
\text { Arthroses } \\
\text { rhumatoïdes } \\
10 \% \text { autres }\end{array}$ & $\begin{array}{l}\text { Révision de } \\
\text { prothèses (risque x8) } \\
\text { Arthroses } \\
\text { rhumatoïdes (risque } \\
\text { x 2.6) }\end{array}$ & réalisé & $\mathrm{Nr}$ & $\begin{array}{l}\text { Incidence: } 1.3 \% \\
49 \% \text { précoce } \\
32 \% \text { tardive } \\
10 \% \text { incertains } \\
50 \% \text { Staph aureus } \\
9.6 \% \text { Staph. epidermidis, } \\
\text { Diphtheroids } \\
15 \% \text { Str group B } \\
\text { 1.9\% Lactobacillus } \\
5.7 \% \text { Klebsiella, } \\
\text { Pseudomonas } \\
\text { 7.6\% Escherichia coli }\end{array}$ & $\begin{array}{l}\text { Quelques jours- 5ans } \\
4 \text { mois- } 9 \text { ans }\end{array}$ & Gingivite \\
\hline $\begin{array}{l}\text { Schmalzried } \\
\text { et al. }[57]^{2}\end{array}$ & $\begin{array}{l}43(47 \\
\text { prothèses } \div \\
\text { en } 3 \text { groupes } \\
\text { selon la date } \\
\text { de pose) }\end{array}$ & $\begin{array}{l}50 \text { ans } \\
\text { (contamination } \\
\text { chirurgicale) } \\
49 \text { ans } \\
\text { (hématogènes) } \\
47 \text { ans } \\
\text { Infection } \\
\text { récurrente } \\
53 \% \mathrm{~F} \\
47 \% \mathrm{H}\end{array}$ & $\begin{array}{l}\text { PTH } \\
15 \% \\
\text { ostéoarthroses } \\
13 \% \\
\text { ostéonécroses } \\
11 \% \text { Arthroses } \\
\text { rhumatödes } \\
\text { 4\% dysplasie } \\
\text { congénitale de la } \\
\text { hanche }\end{array}$ & $\begin{array}{l}\text { Maladies } \\
\text { systémique: } \\
38 \% \text { contamination } \\
\text { chirurgicale } \\
74 \% \text { hématogènes } \\
38 \% \text { infections } \\
\text { récurrentes }\end{array}$ & $\mathrm{Nr}$ & $\mathrm{Nr}$ & $\begin{array}{l}\text { Incidence } 1.5 \% \\
0.4 \% \text { contamination péri- } \\
\text { opératoire } \\
0.6 \% \text { hématogènes } \\
0.4 \% \text { infections } \\
\text { récurrentes } \\
\text { 33\% Staph aureus } \\
16 \% \text { Escherichia coli } \\
13 \% \text { Staph epidermidis, } \\
\text { Pseudomonas } \\
6 \% \text { polymicrobiennes } \\
\end{array}$ & $\begin{array}{l}\text { moyenne } 20 \text { mois } \\
\qquad(1-60) \\
\text { moyenne } 40 \text { mois }(1-96) \\
\text { moyenne } 42 \text { mois }(1-84)\end{array}$ & 1 patient $(\mathrm{Nr})$ \\
\hline $\begin{array}{l}\text { Uçkay et al. } \\
\text { [58] }\end{array}$ & 6101 & $\begin{array}{l}69.9 \text { ans } \\
\mathrm{Nr}\end{array}$ & $\begin{array}{l}66 \% \text { PTH } \\
34 \% \text { PTG } \\
7 \% \text { révision }\end{array}$ & $\begin{array}{l}\text { Tumeur maligne } \\
\text { Diabète sucré } \\
\text { alcoolisme }\end{array}$ & $\mathrm{Nr}$ & $\mathrm{Nr}$ & $\begin{array}{l}\text { Incidence: } 1.2 \% \\
\text { (1.1\%PTH/ } 1.2 \% \mathrm{PTG}) \\
10 \% \text { hématogènes }\end{array}$ & $\begin{array}{l}\text { Moyenne } 33 \text { mois } \\
\text { (6-67) }\end{array}$ & Abcès dentaires ( 3 cas) \\
\hline
\end{tabular}




\begin{tabular}{|c|c|c|c|c|c|c|c|c|c|}
\hline & & & $\begin{array}{l}93 \% \text { première } \\
\text { arthroplastie } \\
\text { Polymyalgie } \\
\text { Arthroses } \\
\text { rhumatoïdes } \\
\text { lupus } \\
\text { érythémateux }\end{array}$ & $\begin{array}{l}\text { Insuffisance rénale } \\
\text { IMC } \\
\text { ASA 3-4 } \\
\text { révision }\end{array}$ & & & $\begin{array}{l}29.6 \% \text { précoces } \\
33.8 \% \text { retardées: } 28.6 \% \\
\text { hématogènes } \\
36.6 \% \text { tardives: } 71.4 \% \\
\text { hématogènes } \\
42 \% \text { Escherichia coli } \\
8.6 \% \text { Staph aureus, } \\
\text { Klebsiella } \\
5 \% \text { Bacteroides fragilis, } \\
\text { Str pneumoniae } \\
\text { 3.7\% Enterococcus } \\
\text { faecalis, Proteus } \\
\text { mirabilis, Str bovis } \\
1.2 \% \text { Str oralis }\end{array}$ & $\begin{array}{l}<3 \text { mois } \\
>24 \text { mois }\end{array}$ & \\
\hline $\begin{array}{l}\text { Waldman et } \\
\text { al. [59] }\end{array}$ & $9^{*}$ & $\begin{array}{l}65 \text { ans } \\
(56-76) 67 \% \mathrm{~F} \\
33 \% \mathrm{H}\end{array}$ & $\begin{array}{l}\text { PTG } \\
22 \% \text { Arthroses } \\
\text { rhumatoïdes } \\
44 \% \\
\text { ostéoarthroses }\end{array}$ & $\begin{array}{l}33 \% \text { diabète sucré } \\
22 \% \text { corticothérapie }\end{array}$ & $\mathrm{Nr}$ & $\mathrm{Nr}$ & $\begin{array}{l}\text { Incidence: } \\
2.1 \% \\
\text { Infection tardive } \\
\text { profonde } \\
33 \% \text { Str viridans } \\
22 \% \text { Peptococcus } \\
11 \% \text { Staph aureus Mr, Str } \\
\text { mutans, Serratia } \\
\text { marcescens }\end{array}$ & $\begin{array}{l}\text { Moyenne } 72 \text { mois } \\
\text { (26-95) }\end{array}$ & $\begin{array}{l}\text { Parodontites } \\
\text { Abcès parodontaux }\end{array}$ \\
\hline
\end{tabular}

*12 patients infectés sur 604 patients (801 prothèses)

**9 patients ayant subis des traitements dentaires en rapport avec l'infection compris dans les 3490 PTG posées, dont 74 PTG infectées

Nr: non renseigné, H: Homme, F: femme, PTH: prothèse totale de hanche, PTG : prothèse totale de genou, PTE: prothèse totale d'épaule, PTC: prothèse totale de coude str: Streptococcus, staph: Staphylococcus, Mr: Methicillin resistant, IMC: indice de masse corporelle 
Table 4: Evaluation qualitative de l'étude en utilisant l'échelle Newcastle-Ottawa pour les études de cohortes.

\begin{tabular}{|c|c|c|c|c|c|c|c|c|c|}
\hline \multirow[t]{2}{*}{ Article, Année } & \multicolumn{4}{|c|}{ Sélection } & \multirow{2}{*}{$\begin{array}{l}\text { Comparabilité } \\
\text { des cohortes } \\
\text { (adaptées pour) }\end{array}$} & \multicolumn{3}{|c|}{ Résultat } & \multirow{2}{*}{$\begin{array}{l}\text { Score } \\
\text { total }\end{array}$} \\
\hline & $\begin{array}{l}\text { Représentativité des } \\
\text { cohortes exposées }\end{array}$ & $\begin{array}{l}\text { Sélection } \\
\text { des } \\
\text { cohortes } \\
\text { non } \\
\text { exposées }\end{array}$ & $\begin{array}{l}\text { Vérification de } \\
\text { l'exposition }\end{array}$ & $\begin{array}{l}\text { Résultat } \\
\text { non présent } \\
\text { au départ }\end{array}$ & & $\begin{array}{l}\text { Evaluation } \\
\text { du résultat }\end{array}$ & $\begin{array}{l}\text { Durée du } \\
\text { suivi } \\
\text { suffisante }\end{array}$ & $\begin{array}{l}\text { Suivi } \\
\text { adéquat }\end{array}$ & \\
\hline Andrews [48] 1981 & - & - & - & - & - & Q & T & Q & 3 \\
\hline Aomori [49] 2003 & - & - & ( & $\square$ & - & ( & $\square$ & - & 4 \\
\hline Barrington [50] 2011 & - & - & Q & $\square$ & - & ( & - & - & 3 \\
\hline Grogan [51]1986 & - & - & - & $\square$ & - & $\square$ & $\square$ & ( & 4 \\
\hline Hamilton [52] 2008 & - & - & - & $\square$ & - & ( & $\square$ & - & 3 \\
\hline Jacobsen [53] 1980 & - & - & - & $\square$ & - & $\square$ & $\square$ & - & 3 \\
\hline Lampley [54] 2014 & - & - & $\square$ & $\square$ & - & 0 & $\square$ & $\square$ & 5 \\
\hline Maderazo [55] 1988 & - & - & - & Q & - & $\square$ & $\square$ & - & 3 \\
\hline Poss [56] 1984 & - & - & - & - & - & $\overline{0}$ & 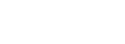 & Q & 3 \\
\hline $\begin{array}{l}\text { Schmalzried [57] } \\
1992\end{array}$ & - & - & - & - & - & 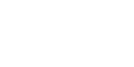 & 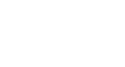 & ( & 3 \\
\hline Uçkay [58] 2009 & - & - & - & $\square$ & - & $\square$ & $\square$ & ( & 4 \\
\hline Waldman [59] 1997 & - & - & - & $\square$ & - & $\square$ & $\square$ & ( & 4 \\
\hline
\end{tabular}

NB: l'évaluation de la qualité est réalisée selon l'objectif de la revue systématique, et peut ne pas refléter la validité interne de chaque étude spécifique. 
Table 5: Evaluation qualitative de l'étude en utilisant l'échelle Newcastle-Ottawa pour les études cas témoins

\begin{tabular}{|c|c|c|c|c|c|c|c|c|}
\hline $\begin{array}{l}\text { Article, } \\
\text { Année }\end{array}$ & $\begin{array}{l}\text { Définition } \\
\text { adéquat } \\
\text { des cas }\end{array}$ & $\begin{array}{l}\text { Représentativité } \\
\text { des cas }\end{array}$ & $\begin{array}{l}\text { Sélection } \\
\text { des } \\
\text { contrôles }\end{array}$ & $\begin{array}{l}\text { Contrôle pour } \\
\text { facteur } \\
\text { important ou } \\
\text { facteur } \\
\text { supplémentaire }\end{array}$ & $\begin{array}{l}\text { Evaluation } \\
\text { de } \\
\text { exposition }\end{array}$ & $\begin{array}{l}\text { Même } \\
\text { méthode de } \\
\text { constatation } \\
\text { pour les cas et } \\
\text { les contrôles }\end{array}$ & $\begin{array}{l}\text { Taux de } \\
\text { non } \\
\text { réponse }\end{array}$ & $\begin{array}{l}\text { Score } \\
\text { total }\end{array}$ \\
\hline $\begin{array}{l}\text { Peersman [3] } \\
2001\end{array}$ & $\overline{0}$ & $\overline{0}$ & $\overline{0}$ & - & - & - & - & 3 \\
\hline $\begin{array}{l}\text { Kessler [45] } \\
2012\end{array}$ & प & प & Q & - & - & - & - & 3 \\
\hline $\begin{array}{l}\text { Kaandorp } \\
\text { [46] } 1995\end{array}$ & - & प & - & ( & - & - & - & 2 \\
\hline $\begin{array}{l}\text { Cordero- } \\
\text { Ampuero } \\
\text { [47] } 2010\end{array}$ & प & प & प & - & - & - & - & 3 \\
\hline
\end{tabular}

NB: l'évaluation de la qualité est réalisée selon l'objectif de la revue systématique, et peut ne pas refléter la validité interne de chaque étude spécifique. 
Articles identifiés dans les bases de données $(n=3179)$

3095 articles exclus sur titre et résumé

Exclusion selon critères sélection $n=54$

Pas d'accès au texte entier $n=1$

Pas de traduction possible $n=1$

28 articles inclus 